\title{
In-situ high-temperature emissivity spectra and thermal expansion of $C 2 / c$ pyroxenes: Implications for the surface of Mercury
}

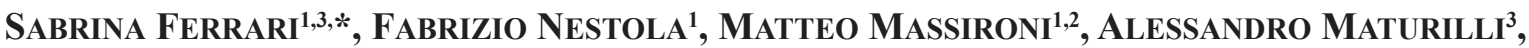 Jörn Helbert ${ }^{3}$, MATteo Alvaro ${ }^{1,4}$, M. Chiara DomeneghetTI ${ }^{5}$ ANd Federico Zorzi ${ }^{1}$}

\author{
${ }^{1}$ Department of Geosciences, University of Padua, Via G. Gradenigo 6, 35131 Padova, Italy \\ ${ }^{2}$ Astronomical Observatory of Padua, INAF, Vicolo Osservatorio 5, 35122 Padova, Italy \\ ${ }^{3}$ Institute for Planetary Research, DLR, Rutherfordstrasse 2, 12489 Berlin-Adlershof, Germany \\ ${ }^{4}$ IRSPS, G. D’Annunzio University, Via Pindaro 42, 65127 Pescara, Italy \\ ${ }^{5}$ Department of Earth and Environmental Sciences, University of Pavia, Via Ferrata 1, 27100 Pavia, Italy
}

\begin{abstract}
This work was carried out within the framework of the European Space Agency and Japanese Aerospace Exploration Agency BepiColombo space mission to Mercury and intends to provide valid tools for the interpretation of spectra acquired by the MErcury Radiometer and Thermal Infrared Spectrometer (MERTIS) on board of BepiColombo.

Two $C 2 / c$ augitic pyroxenes, with different $\mathrm{Mg} / \mathrm{Fe}$ ratios and constant $\mathrm{Ca}$ contents, were investigated by in situ high-temperature thermal infrared spectroscopy and in situ high-temperature single-crystal X-ray diffraction up to temperatures of about 750 and $770 \mathrm{~K}$, respectively.

The emissivity spectra of the two samples show similar band center shifts of the main three bands toward lower wavenumbers with increasing temperature. In detail, with increasing temperature bands 1 and 2 of both samples show a much stronger shift with respect to band 3, which remains almost unchanged. Our results indicate that the center positions of bands 1 and 2 are strong functions of the temperature, whereas the center position of band 3 is a strong function of the $\mathrm{Mg} \#$ [with $\mathrm{Mg} \#=\mathrm{Mg}$ / $\left(\mathrm{Mg}+\mathrm{Fe}^{2+}\right)$ atomic ratio].

The analysis of the thermal behavior gives similar thermal expansion volume coefficients, $\alpha_{\mathrm{v}}$, for the Mg-rich and Fe-rich samples, with $\alpha_{\mathrm{V}}=2.72(8)$ and $2.72(7) \times 10^{-5} \mathrm{~K}^{-1}$, respectively, using the Berman (1988) equation. This correspondence totally explains the band center shifts similarity between the two samples.

Our data suggest that MERTIS spectra will be able to provide indications of $C 2 / c$ augitic pyroxene $\mathrm{Mg \#}$ and will allow a correct interpretation that is independent on the spectra acquisition temperature.

Keywords: High temperature, TIR emissivity, thermal expansion, clinopyroxenes, Mercury
\end{abstract}

